\title{
Total or subtotal glossectomy with laryngeal preservation : a national study of 29 patients
}

\section{Keski-Säntti, Harri}

2018-01

Keski-Säntti , H , Bäck , L , Lassus , P , Koivunen , P , Kinnunen , I, Blomster , H , Mäkitie , A A \& Aro , K 2018 , ' Total or subtotal glossectomy with laryngeal preservation : a national study of 29 patients ' , European Archives of Oto-Rhino-Laryngology , vol. 275 , no. 1 , pp. 191-197 . https://doi.org/10.1007/s00405-017-4789-z

http://hdl.handle.net/10138/298406

https://doi.org/10.1007/s00405-017-4789-z

publishedVersion

Downloaded from Helda, University of Helsinki institutional repository.

This is an electronic reprint of the original article.

This reprint may differ from the original in pagination and typographic detail.

Please cite the original version. 


\title{
Total or subtotal glossectomy with laryngeal preservation: a national study of 29 patients
}

\author{
Harri Keski-Säntti ${ }^{1}\left[\right.$ - Leif Bäck ${ }^{1} \cdot$ Patrik Lassus $^{2} \cdot$ Petri Koivunen $^{3}$ • \\ Ilpo Kinnunen ${ }^{4}$ Henry Blomster ${ }^{5}$ Antti A. Mäkitie ${ }^{1} \cdot$ Katri Aro $^{1}$
}

Received: 22 August 2017 / Accepted: 24 October 2017 / Published online: 8 November 2017

(c) Springer-Verlag GmbH Germany 2017

\begin{abstract}
Total glossectomy remains a controversial procedure as it often leads to notorious sequalae in swallowing and speaking functions. Disease entities indicating total glossectomy tend to have poor prognosis. We evaluated whether this type of surgery can be concidered justified based on our national series. We reviewed all total and subtotal glossectomies with laryngeal preservation performed in Finland between 2005 and 2014 in terms of overall survival (OS), disease-specific survival (DSS), locoregional control (LRC), and functional outcome as assessed by gastric tube or tracheostomy dependence and ability to produce intelligible speech. Of the 29 eligible patients, 15 had undergone total and 14 subtotal glossectomy with curative intent. In eight patients, total/subtotal glossectomy was performed as salvage procedure after the previous treatment. One-year estimates for OS, DSS, and LRC were 48, 59, and 66\%, and corresponding 3-year estimates were 31,46 , and $46 \%$, respectively. The gastrostomy and tracheostomy dependence rates at 1 year after operation were 77 and 15\%, respectively. Fifty-nine percent of the patients were assessed to be able
\end{abstract}

Harri Keski-Säntti

harri.keski-santti@hus.fi

1 Department of Otorhinolaryngology, Head and Neck Surgery, Helsinki University Hospital and University of Helsinki, P.O.Box 263, 00029 HUS Helsinki, Finland

2 Department of Plastic Surgery, Helsinki University Hospital and University of Helsinki, Helsinki, Finland

3 Department of Otorhinolaryngology, Head and Neck Surgery, Oulu University Hospital, Oulu, Finland

4 Department of Otorhinolaryngology, Head and Neck Surgery, Turku University Hospital, Turku, Finland

5 Department of Otorhinolaryngology, Head and Neck Surgery, Kuopio University Hospital, Kuopio, Finland to communicate verbally. As in most other published studies, we found unsatisfactory survival figures after subtotal or total glossectomy and most patients remained dependent on gastrostomy tube. This surgery is, however, presumably the best and often only chance for cure in a selective patient population, and according to our opinion, it is indicated as a primary or salvage treatment provided that the reconstruction is planned optimally to guarantee a reasonable quality of life after surgery.

Keywords Glossectomy · Tongue reconstruction · Tongue cancer · Oral cancer · Survival · Quality of life

\section{Introduction}

Total glossectomy remains a controversial procedure. In most centres, the treatment of choice for advanced oral cavity cancer is primary surgery and postoperative radiotherapy (RT) or chemoradiotherapy (CRT). However, some consider primary total glossectomy unacceptable because of related high morbidity [1]. Traditionally, total or subtotal glossectomy with laryngeal preservation has shown poor functional results as many patients have remained dependent on gastrostomy tube. It has even been suggested that total laryngectomy should routinely be combined with total glossectomy to prevent aspiration. However, as reconstruction techniques have evolved, also better functional results have recently been reported in some small series. For example, in a study by Longo et al., all 13 patients resumed oral feeding after total glossectomy with laryngeal preservation [2].

Survival figures after total glossectomy have remained unsatisfactory. According to a review by Varthanian et al., the 3-year overall survival (OS) after total or subtotal glossectomy rarely exceeds $35 \%$ [3]. In some recent studies, 
definitive concomitant CRT has been used for advanced oral cavity tumours with encouraging survival and functional outcomes and thus, sparing many patients from total glossectomy $[4,5]$. However, also opposite results have been published favouring surgery as a primary treatment modality for advanced oral cancer [6, 7]. Prospective randomized trials comparing surgery followed by RT or CRT with definitive concomitant CRT have not been published.

According to the national guidelines for the management of head and neck cancer in Finland, the preferred treatment for oral cancer is surgery \pm adjuvant RT or CRT. Even extensive oral cavity tumours necessitating total or subtotal glossectomy are usually primarily operated. Base of tongue tumours is usually treated by RT or CRT with surgery as a salvage option, although the emergence of transoral robotic surgery provides options for this treatment algorithm. In the current study, we aimed to retrospectively assess the survival and functional outcome of patients who underwent subtotal or total glossectomy in a nation-wide series.

\section{Patients and methods}

Institutional research approvals to conduct this retrospective national multicenter study were obtained ( $§ 191$, December, 30, 2014). This research involved only patient charts and, therefore, no formal Research Ethics Board approval or informed consent was needed according to the Finnish legislation. Clinicopathological data of all patients who had total or subtotal glossectomy performed at the five university hospitals in Finland during the 10-year period between 2005 and 2014 were reviewed. Subtotal glossectomy was defined as complete removal of oral tongue at minimum and total glossectomy as complete removal of tongue down to vallecula on both sides. Patients who, in addition to glossectomy, had any supraglottic laryngeal structures removed were included in the study. Patients who, in addition to glossectomy, had total laryngectomy and thereby permanent aerodigestive separation performed were excluded.

This study represents national data, as total or subtotal glossectomies are performed only at the five university hospitals in Finland with a population of 5.4M. All patients had been evaluated by a local multidisciplinary tumour board. The hospital records were reviewed and data on patient and tumour characteristics, treatment, and follow-up were recorded. Pre-treatment evaluation consisted of full medical history, clinical examination and tumour biopsy, imaging of the primary site and neck by CT or MRI, imaging of chest and upper abdomen by CT, and dental evaluation. To acquire additional support for postoperative function, all patients were referred to an institutional speech and swallowing therapist as well as to a physiotherapist postoperatively. Functional evaluation in terms of swallowing and breathing was performed by assessing gastrostomy tube and tracheostomy dependence or independence. Data on patients' ability to produce intelligible speech were recorded from the patient charts.

\section{Follow-up}

During the first year of follow-up, patients were seen every 2-3 months, then during the second year every 3-4 months, and thereafter every 4-6 months. According to our national protocol, follow-up is continued until 5 years from the completion of treatment or last episode of recurrent disease.

\section{Statistical analysis}

Statistical analyses were conducted using the NCSS software (NCSS 8, LLC. Kaysville, Utah, USA. http://www. ncss.com). We report values as medians with range for descriptive purposes. Survival rates were calculated using the Kaplan-Meier method and the survival functions were compared using the log-rank test. The Cox proportional hazards model was used to estimate the prognostic value of clinical variables, including minimal pathological marginal of the primary tumour specimen, $\mathrm{pN}$ class, extranodal extension, postoperative RT vs. CRT, and Charlson Comorbidity Index (CCI). The multivariate Cox regression analysis could not be used because of the high amount of variables compared with the patient number. In addition, Fisher's exact test was used to test the differences between percutaneous endoscopic gastrostomy (PEG) tube dependencies. All statistical tests were two-sided, and $\mathrm{P}$ values less than 0.05 were considered statistically significant.

\section{Results}

Twenty-nine patients were eligible for inclusion, and there were 20 males and 9 females. The median age was 62 years (range 28-88). The median follow-up time was 10 months for all patients (range 0-118) and 32 months for those alive (range 13-118). Twenty-one patients had been operated at the Helsinki University Hospital, and three, one, four, and zero patients at the University Hospitals of Oulu, Turku, Kuopio, and Tampere, respectively. None of the patients presented with distant metastases at the time of surgery, which was in all cases performed with curative intent. The distribution of the primary tumour site was as follows: oral tongue, $n=21(72 \%)$; base of tongue, $n=3(10 \%)$; floor of mouth, $n=5(17 \%)$. Almost all tumours $(n=28)$ were epidermoid carcinomas and one was adenoid cystic carcinoma. Twenty-one (72\%) patients had subtotal/total glossectomy as primary treatment, while eight $(28 \%)$ patients had been 
treated previously. A prophylactic PEG tube was placed and a tracheostomy was performed for all patients.

Total glossectomy was performed in 15 patients and subtotal glossectomy in 14 patients. Free flaps were used to reconstruct the glossectomy defect in 27 patients and pedicular flaps in two patients. The most common reconstruction was anterolateral thigh flap (ALT, $n=18$ ) followed by six other types of flaps. Total flap loss occurred in three patients, which were all successfully salvaged with pedicled pectoralis major flap in two patients and a new ALT flap in one patient. The resection included supraglottic structures in two patients: one partial resection of epiglottis and one supraglottic laryngectomy. Neck dissection was performed bilaterally in 23 patients, and ipsilaterally in five patients. One patient without neck dissection had had previous surgical treatment including neck dissection. Metastatic lymph nodes were detected in 17 (59\%) patients, including extranodal extension in 12 patients $(71 \%)$. The histopathological margins were close or positive $(<5 \mathrm{~mm})$ in 18 patients. More data are given in Table 1.

In 21 patients (72\%), glossectomy was performed as the primary treatment, and in eight patients $(28 \%)$ as salvage treatment. The previous treatment included RT or CRT in six cases and surgery in addition to oncological treatment in two cases. Postoperative RT (intensity modulated) was scheduled for nine patients and CRT for eight patients. Two of these 17 patients failed to complete adjuvant treatment because of poor general health or death during treatment.

Table 1 Surgical treatment of the 29 patients

\begin{tabular}{lll}
\hline & $n$ & $\%$ \\
\hline Glossectomy type (subtotal/total) & $14 / 15$ & $48 / 52$ \\
Supraglottic resection (yes/no) & $2 / 27$ & $7 / 93$ \\
Mandibular resection (no/rim/segmental) & $23 / 5 / 1$ & $79 / 17 / 3$ \\
Access mandibulotomy (yes/no) & $6 / 23$ & $21 / 79$ \\
Hyoid suspension (yes/no) & $3 / 26$ & $10 / 90$ \\
Motor innervation (yes/no) & $6 / 23$ & $21 / 79$ \\
Neck dissection (no/ipsilateral/bilateral) & $1 / 7 / 21$ & $3 / 24 / 83$ \\
Flap used & & \\
ALT & 18 & 62 \\
LD & 5 & 17 \\
VRAM & 2 & 7 \\
RFA & 1 & 3 \\
TMG & 1 & 3 \\
LD (pedicular) & 1 & 3 \\
Pectoralis major & 1 & 3 \\
Flap loss (yes/no) & $3 / 26$ & $10 / 90$ \\
Close or positive margin $(<5$ mm) (yes/no) & $18 / 11$ & $62 / 38$ \\
\hline
\end{tabular}

$A L T$ anterolateral thigh flap, $L D$ latissimus dorsi flap, $V R A M$ vertical rectus abdominis myocutaneous flap, $R F A$ radial forearm flap, $T M G$ transverse myocutaneus gracilis flap
Therefore, 15 patients completed postoperative RT; seven of them with concurrent cisplatin-based chemotherapy. The median radiation dose was $60 \mathrm{~Gy}$ (range 56-70).

\section{Patterns of relapse, survival, and local control}

Thirteen patients experienced a recurrence during their follow-up: local only, $n=5$; regional only, $n=1$; distant only, $n=1$; local and regional, $n=2$; regional and distant, $n=2$; local, regional, and distant, $n=2$. Twelve of these thirteen patients have died of their disease and one patient is alive with locally recurrent, incurable disease.

During the study period, 19 patients $(66 \%)$ died, of which 12 died of the index disease and seven of other causes. The 1 -year estimates for OS, disease-specific survival (DSS), and locoregional control (LRC) were 48, 59, and 66\%, while corresponding 3 -year estimates were 31,46 , and $46 \%$, respectively (Figs. 1, 2, 3).

OS, when analyzed using the Cox proportional hazards regression model, was not significantly related to histopathological free margins of the primary tumour specimen, pN class, extranodal extension, and postoperative RT vs. CRT [hazard ratio (HR) 1.0500-2.101, $P=0.214-0.697$ ]. In univariate analysis, higher CCI (HR 1.391, 95\% CI $0.0108-0.649, \mathrm{P}=0.0428$ ) significantly predicted poor OS.

\section{Gastrostomy and tracheostomy dependence}

In total, during the follow-up, PEG tube was removed for five patients (17\%), of which one died 5 months after PEG removal and the other four have survived. Of the 18 patients alive at 6 months after operation, 15 (83\%) had PEG tube in place. Of the 13 patients alive at 1 year after

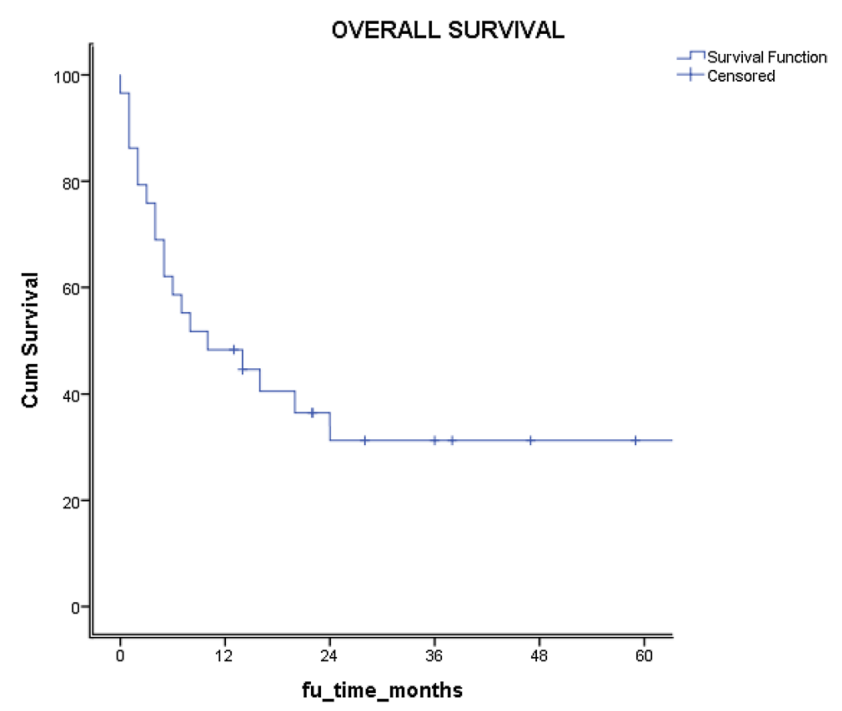

Fig. 1 Overall survival of the whole study group 


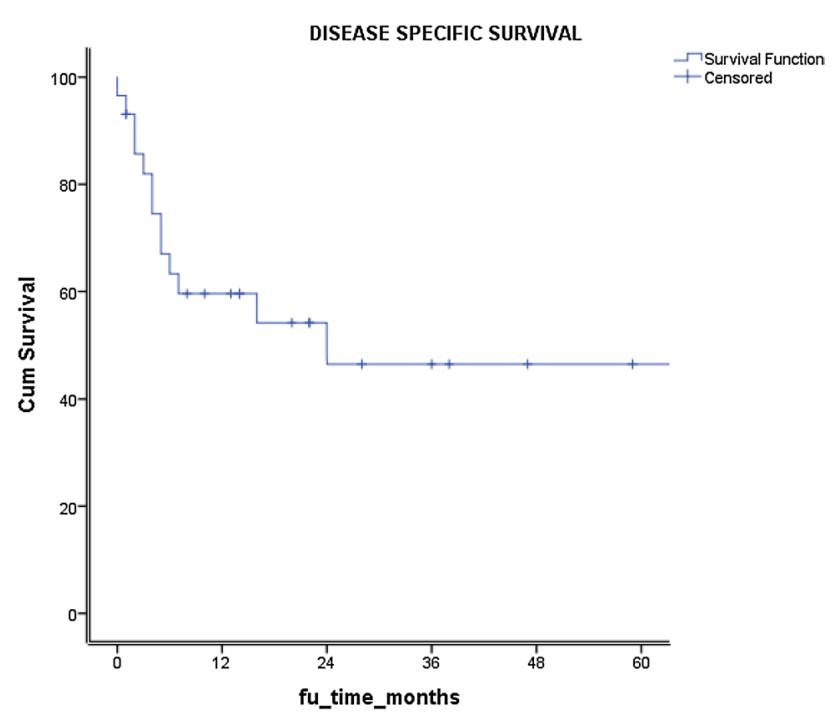

Fig. 2 Disease-specific survival of the whole study group

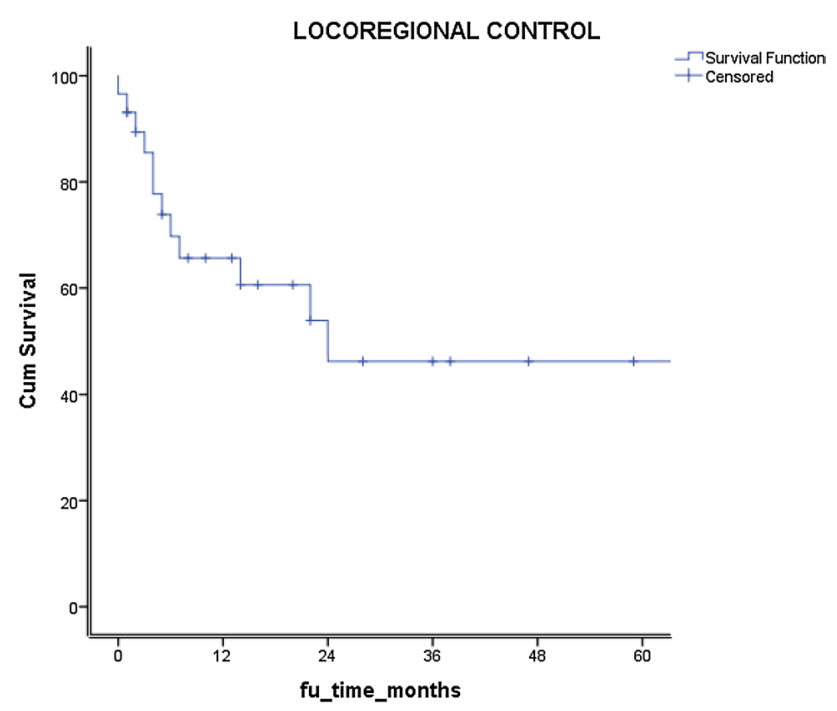

Fig. 3 Locoregional control of the whole study group

surgery, $10(77 \%)$ had PEG tube in place. Differences in PEG tube dependence were not found between the following patient groups: total glossectomy vs. subtotal glossectomy, continuity mandibular resection vs. no resection, access mandibulotomy vs. no mandibulotomy, and ALT flap vs. other fasciocutaneous flaps.

Altogether, 17 patients (59\%) had tracheostomy tube removed. The median time interval from subtotal/total glossectomy to decannulation was 15 days (range 7-655). Of the 13 patients alive at 1 year after operation, 11 had been decannulated (85\%).

\section{Speech}

Verbal communication after surgery was assessed as 'possible' in 17 patients (59\%) and as 'impossible' in 11 (38\%) patients. For one patient (3\%), these data could not be collected retrospectively.

\section{Discussion}

We reviewed 29 patients who had total or subtotal glossectomy performed in Finland between 2005 and 2014. Estimates for OS, DSS, and LRC at 1 year were 48, 59, and $66 \%$ and at 3 years 31,46 , and $46 \%$, respectively. The longterm PEG tube and tracheostomy dependence rates were 77 and $15 \%$, respectively. More than half of the patients were assessed to be able to produce intelligible speech after operation. The survival figures in our study concur with others. According to many published studies, the survival after total glossectomy has remained unsatisfactory and a review by Vartanian et al. concluded, that for this particular patient group, a 5-year OS above $25 \%$ in most series can be considered acceptable [3, 8-11]. In univariate analysis, we found only high CCI to predict poor survival.

We evaluated the functional results only using by PEG tube or tracheostomy dependence as rough estimates. Of the 13 patients who survived for at least 1 year, $10(77 \%)$ had PEG tube in place at 1 year after operation. Therefore, only three $(23 \%)$ of these patients resumed the ability to maintain body weight by oral feeding only. It seems, however, that patients living with PEG tube are able to handle their secretions as none of them required total laryngectomy due to chronic aspiration. We did not find any predictive factors for poor swallowing. Better figures for swallowing rehabilitation have been published after subtotal or total glossectomy with laryngeal preservation (Table 2 ). In a review by Rigby et al., gastric tube dependence ranged from 30 to $44 \%$ [12]. Laryngeal suspension, i.e., circumhyoid sutures suspending the larynx from the mandibular bone, recommended by many authors to facilitate swallowing function [12-18], was performed in our series only in three patients and they all remained PEG tube dependent. Segmental mandibular resection has been connected with impaired functional results [8]. In our study group, however, only one patient underwent segmental mandibulectomy. Total glossectomy as a salvage procedure after the previous RT or CRT shows worse functional results $[12,17]$. In the present series, however, this explanation for poor swallowing was unclear as only eight patients had had previous treatment. In a study by Miyamoto et al., advanced age ( $\geq 70$ years), being underweight (BMI < 18.5), and, most importantly, postoperative RT were identified as risk factors for gastrostomy tube dependence following tongue reconstruction [19]. Their study included 
Table 2 Studies assessing functional results of total/subtotal glossectomy with laryngeal preservation

\begin{tabular}{|c|c|c|c|c|c|c|c|}
\hline Study & Study design & $n$ & $\begin{array}{l}\text { Primary treat- } \\
\text { ment, } n(\%)\end{array}$ & Flap(s) used most often, $(n)$ & $\begin{array}{l}\text { Decannula- } \\
\text { tion, }(\%)\end{array}$ & $\begin{array}{l}\text { Feeding tube, } \\
\text { removed }(\%)\end{array}$ & $\begin{array}{l}\text { Intelligable } \\
\text { speech }(\%)\end{array}$ \\
\hline Yanai et al. [24] & Retrospective & 17 & 17 (100) & RAM (11) & 100 & 82 & 82 \\
\hline Dziegielewski et al. [25] & Prospective & 12 & $12(100)$ & $\operatorname{ALT}(12)$ & 92 & 50 & 100 \\
\hline Rihani et al. [17] & Retrospective & 94 & $36(38)$ & Pectoralis (35), RAM (32) & 84 & 29 & ND \\
\hline Navach et al. [11] & Retrospective & 37 & $24(65)$ & ALT (20), Gracilis (13) & ND & 70 & 100 \\
\hline Sinclair et al. [10] & Retrospective & 20 & $9(45)$ & RAM (16) & 50 & 0 & 30 \\
\hline Leymarie et al. [21] & Retrospective & 10 & ND & $\operatorname{ALT}(10)$ & 100 & 100 & 80 \\
\hline Longo et al. [2] & Prospective & 13 & ND & ALT (13) & 100 & 100 & 100 \\
\hline Paydarfar et al. [13] & Retrospective & 8 & ND & ALT (8) & 100 & 75 & 100 \\
\hline
\end{tabular}

$R A M$ rectus abdominis myocutaneus, $A L T$ anterolateral thigh flap, $N D$ not defined

different kinds of tongue reconstructions and opposite to many other reports; surprisingly, the extent of resection (i.e., subtotal or total glossectomy vs. less extensive resections) did not significantly predict gastrostomy tube dependence. The authors speculate that this finding can be attributed to recent technical advances in tongue reconstruction. The long-term tracheostomy dependence rate of $15 \%$ in our series is in line with other studies. A review by Rigby et al. concluded that between 85 and $95 \%$ of patients are able to have their tracheostomy tube removed after total glossectomy without laryngectomy [12].

In the present study, total/subtotal glossectomy was performed as a salvage procedure after the previous treatment in eight patients. Seven of these patients died, i.e., four died of the index disease and three of other causes. This is in accordance with the previous studies, in which both the survival and functional outcome of the previously treated patients have been inferior to that of patients undergoing primary treatment $[11,17,20]$.

As in our material, anterolateral thigh-free flap (ALT) and vertical rectus abdominis myocutaneous-free flap (VRAM) are the flaps most often used to reconstruct total glossectomy defects $[2,8,9,11,16-18]$. To optimize functional results, several technical aspects have been proposed. Most importantly, the reconstructed neotongue needs to have sufficient tissue bulk to allow contact with the palate, which is thought to be crucial for both speech and swallowing functions. Leymarie et al. have introduced a cathedral triptych shape ALT flap that provides close to normal shape and volume of the neotongue with good functional results [21]. Kimata et al. demonstrated that there is a correlation between flap shape and patients' ability to speak and swallow. In their study, flaps classified as protuberant were associated with improved functional outcome [16]. Yun et al. have showed similar benefit with increased flap volume [15]. As we have experienced in our patients, they also showed that the flap volume decreases over time leading to impaired swallowing function and speech intelligibility. In their study, more considerable loss of volume was noticed in ALTs as compared with VRAMs. Furthermore, they recommend to consider a functional muscle to prevent muscle atrophy in the flap, and laryngeal suspension to reduce aspiration, and facilitate swallowing. Laryngeal suspension is also recommended in numerous other studies [12-14, 16-18]. Reinnervation of the flap failed to show importance in a study by $\mathrm{Yu}$ and Robb, especially in patients undergoing postoperative RT, because RT seems to induce severe fibrosis in the flap muscle compromising the benefits of motor reinnervation. Instead, they recommend sensory reinnervation, which they found to result in better swallowing function [18]. This aspect is supported in a study by Chang et al. including improved speech [8]. In a study by Rihani et al., 94 patients undergoing total glossectomy with laryngeal preservation were reconstructed using various flaps, but they found no difference in the functional outcome between different flaps and concluded that flap selection should be patient specific. They also pointed out that pedicled pectoralis' major myocutaneous flap is still a viable option in reconstruction of total glossectomy defect [17]. Also, in a study by Lin et al., feeding tube dependence was not associated with the type of flap used or flap innervation [22]. It is, however, generally accepted that, from a functional point of view, a RFFF does not allow a bulky enough reconstruction for a total glossectomy defect [18]. We conclude based on our experience that the flap selection should be patient specific, and ALT being usually the first choice. The flap should be designed to have a projective shape and to be large enough to compensate for the subsequent shrinking of the flap. If the patient has any functional tongue left, shaping the neotongue as described by Leymarie et al. might benefit the patient. However, in cases of total loss of function, the patient may benefit from a more simple shape of the neotongue to facilitate the drainage of saliva from the oral cavity.

In contradiction to advanced stage pharyngeal and laryngeal cancer, where definitive concomitant CRT has widely replaced surgical treatment as a primary treatment 
option, surgery remains the primary treatment for oral cavity cancer in most centres. Despite the progress in the field of oncology for head and neck tumours, clinical trials utilizing CRT as primary treatment for oral cavity cancer are scarce. No prospective studies compare primary surgical approach with definitive CRT protocol. In studies by Stenson et al. and McDowell et al., patients with advanced oral cavity tumours who had been treated with definitive CRT were compared in a subset of patients who had had primary surgery. In both studies, there was no difference in survival between the groups, but the functional outcome was better among the oncologically treated patients $[4$, 5]. Opposite results have also been published; however. Crombie et al. recommended primary surgery for oral cavity cancer after having reviewed 54 non-surgically treated patients with oral cancer. They found unsatisfactory survival figures (DSS 30\%) and high rate of osteoradionecrosis (36\% of the surviving patients) [7]. Also a study by Gore et al. ended up in recommending primary surgical treatment [6]. They retrospectively compared two cohorts of oral cancer patients treated either with definitive concomitant CRT or with primary surgery and postoperative RT or CRT. Overall survival and DSS were significantly higher in the surgically treated group with a $94 \%$ reduction in DSS in favor of primary surgery. Takayama et al. have recently published a paper, in which proton beam therapy combined with intra-arterial chemotherapy was used to definitely treat patients diagnosed with stage IIIIVB tongue cancer and the results were very promising; the 3-year OS rate was as high as $87 \%$ [23]. Patients with a very large tongue tumour often have remarkably affected swallowing and speech functions because of the tumour volume, and the possibility to resume these functions after organ sparing treatment must be considered unlikely. Further studies are needed to establish the role of modern CRT protocols in the treatment of advanced oral cavity cancer. Preferably, these should be prospective studies comparing the survival and functional outcome of the two treatment approaches.

Adverse histopathological features were common in the present series, as 18 of our 29 patients had close or positive margins, and 18 had metastatic lymph nodes with extranodal extension in 12 patients. These findings are in concordance with the fact that patients undergoing total glossectomy generally have locoregionally advanced disease resulting in poor survival figures. Sinclair et al. also found the rate of close/ positive margins to be $60 \%$ of total glossectomy patients but only $30 \%$ of patients undergoing total glossolaryngectomy [10]. Regarding patients in the total glossectomy group, $89 \%$ of the close margins occurred at the inferior margin of resection allowing the authors to speculate that total glossolaryngectomy may improve local disease control in patients with advanced squamous cell carcinoma of tongue.
Our study is limited mainly by its retrospective nature and small number of patients. This precludes any meaningful search for predictive factors for survival and functional outcome that would be valuable in clinical decision-making when considering such a mutilating surgery as total glossectomy. The functional results can be assessed retrospectively only in quite a rough manner. Consequently, the present study remains mainly a descriptive analysis of our nationwide clinical practice. We have demonstrated a relatively high rate of PEG tube dependence as compared with some other published series, which encourages us to search for refinement in our surgical technique and to advance supportive care and rehabilitation in swallowing. The most important technical aspects in reconstruction of a glossectomy defect, according to the current literature, are protuberant shape and sufficient tissue bulk enabling contact of the neotongue with the palate even after inevitable shrinkage of the flap, and suspension of the hyoid bone to the mandible. The role of motor and sensory reinnervation of the flap is less clear.

We conclude that survival outcome after subtotal or total glossectomy in our series, as in many other published series, was unsatisfactory with most patients dying during the first year after surgery. There were, however, patients who survived and even a few patients with an excellent functional outcome. Most patients were decannulated and regained the ability to express themselves verbally, but only few of them could have their PEG tube removed. The PEG-dependent patients, however, could handle their secretions and no laryngectomies had to be performed to prevent aspiration. At present, we cannot predict which of the patients with major tongue or floor of mouth tumour will survive. In the absence of prospective clinical trials comparing different treatment modalities, total or subtotal glossectomy is, according to our opinion, definitely a procedure worth performing provided that the reconstruction is planned optimally to guarantee a reasonable functional outcome and quality of life after surgery.

Acknowledgements The authors thank M.Sc. Timo Pessi for performing the statistical analysis for this study.

\section{Compliance with ethical standards}

Conflict of interest The authors declare that they have no conflict of interest.

Ethical approval All procedures performed in studies involving human participants were in accordance with the ethical standards of the institutional and/or national research committee and with the 1964 Helsinki declaration and its later amendments or comparable ethical standards. This research involved only patient charts.

Informed consent As this study was a retrospective chart review, no informed consent was needed according to the Finnish legislation. 


\section{References}

1. Kreeft A, Tan IB, van den Brekel MW, Hilgers FJ, Balm AJ (2009) The surgical dilemma of 'functional inoperability' in oral and oropharyngeal cancer: current consensus on operability with regard to functional results. Clin Otolaryngol 34:140-146

2. Longo B, Pagnoni M, Ferri G, Morello R, Santanelli F (2013) The mushroom-shaped anterolateral thigh perforator flap for subtotal tongue reconstruction. Plast Reconstr Surg 132:656-665

3. Vartanian JG, Magrin J, Kowalski LP (2010) Total glossectomy in the organ preservation era. Curr Opin Otolaryngol Head Neck Surg 18:95-100

4. Stenson KM, Kunnavakkam R, Cohen EE, Portugal LD, Blair E, Haraf DJ, Salama J, Vokes EE (2010) Chemoradiation for patients with advanced oral cavity cancer. Laryngoscope 120:93-99

5. McDowell L, Collins M, Kleid S, Rischin D, Corry J (2014) T4 squamous cell carcinoma of the oral tongue without mandibular involvement: surgery or chemoradiotherapy? Oral Surg Oral Med Oral Pathol Oral Radiol. 117:163-169

6. Gore SM, Crombie AK, Batstone MD, Clark JR (2015) Concurrent chemoradiotherapy compared with surgery and adjuvant radiotherapy for oral cavity squamous cell carcinoma. Head Neck 37:518-523

7. Crombie AK, Farah C, Tripcony L, Dickie G, Batstone MD (2012) Primary chemoradiotherapy for oral cavity squamous cell carcinoma. Oral Oncol 48:1014-1018

8. Chang EI, Yu P, Skoracki RJ, Liu J, Hanasono MM (2015) Comprehensive analysis of functional outcomes and survival after microvascular reconstruction of glossectomy defects. Ann Surg Oncol 22:3061-3069

9. Mazarro A, de Pablo A, Puiggros C, Velasco MM, Saez M, Pamias $\mathrm{J}$, Bescos C (2016) Indications, reconstructive techniques, and results for total glossectomy. Head Neck 38(Suppl 1):E2004-10

10. Sinclair CF, Carroll WR, Desmond RA, Rosenthal EL (2011) Functional and survival outcomes in patients undergoing total glossectomy compared with total laryngoglossectomy. Otolaryngol Head Neck Surg 145:755-758

11. Navach V, Zurlo V, Calabrese L, Massaro MA, Bruschini R, Giugliano G, Ansarin M, Chiesa F (2013) Total glossectomy with preservation of the larynx: oncological and functional results. Br J Oral Maxillofac Surg 51:217-223

12. Rigby MH, Hayden RE (2014) Total glossectomy without laryngectomy - a review of functional outcomes and reconstructive principles. Curr Opin Otolaryngol Head Neck Surg 22:414-418

13. Paydarfar JA, Freed GL, Gosselin BJ (2016) The anterolateral thigh fold-over flap for total and subtotal glossectomy reconstruction. Microsurgery 36:297-302

14. van Lierop AC, Basson O, Fagan JJ (2008) Is total glossectomy for advanced carcinoma of the tongue justified? S Afr J Surg 46:22-25
15. Yun IS, Lee DW, Lee WJ, Lew DH, Choi EC, Rah DK (2010) Correlation of neotongue volume changes with functional outcomes after long-term follow-up of total glossectomy. J Craniofac Surg 21:111-116

16. Kimata Y, Sakuraba M, Hishinuma S, Ebihara S, Hayashi R, Asakage T, Nakatsuka T, Harii K (2003) Analysis of the relations between the shape of the reconstructed tongue and postoperative functions after subtotal or total glossectomy. Laryngoscope 113:905-909

17. Rihani J, Lee MR, Lee T, Ducic Y (2013) Flap selection and functional outcomes in total glossectomy with laryngeal preservation. Otolaryngol Head Neck Surg 149:547-553

18. Yu P, Robb GL (2005) Reconstruction for total and near-total glossectomy defects. Clin Plast Surg 32:411-419, vii

19. Miyamoto S, Sakuraba M, Nagamatsu S, Kayano S, Kamizono K, Hayashi R (2012) Risk factors for gastric-tube dependence following tongue reconstruction. Ann Surg Oncol 19:2320-2326

20. Barry B, Baujat B, Albert S, Nallet E, Depondt J, Guedon C, Gehanno P (2003) Total glossectomy without laryngectomy as first-line or salvage therapy. Laryngoscope 113:373-376

21. Leymarie N, Karsenti G, Sarfati B, Rimareix F, Kolb F (2012) Modification of flap design for total mobile tongue reconstruction using a sensitive antero-lateral thigh flap. J Plast Reconstr Aesthet Surg 65:e169-74

22. Lin DT, Yarlagadda BB, Sethi RK, Feng AL, Shnayder Y, Ledgerwood LG, Diaz JA, Sinha P, Hanasono MM, Yu P, Skoracki RJ, Lian TS, Patel UA, Leibowitz J, Purdy N, Starmer H, Richmon JD (2015) Long-term Functional Outcomes of Total Glossectomy With or Without Total Laryngectomy. JAMA Otolaryngol Head Neck Surg 141:797-803

23. Takayama K, Nakamura T, Takada A, Makita C, Suzuki M, Azami Y, Kato T, Hayashi Y, Ono T, Toyomasu Y, Hareyama M, Kikuchi Y, Daimon T, Mitsudo K, Tohnai I, Fuwa N (2016) Treatment results of alternating chemoradiotherapy followed by proton beam therapy boost combined with intra-arterial infusion chemotherapy for stage III-IVB tongue cancer. J Cancer Res Clin Oncol 142:659-667

24. Yanai C, Kikutani T, Adachi M, Thoren H, Suzuki M, Iizuka T (2008) Functional outcome after total and subtotal glossectomy with free flap reconstruction. Head Neck 30:909-918

25. Dziegielewski PT, Ho ML, Rieger J, Singh P, Langille M, Harris JR, Seikaly H (2013) Total glossectomy with laryngeal preservation and free flap reconstruction: objective functional outcomes and systematic review of the literature, Laryngoscope 123:140-145 